\title{
Pharmacological interventions for treating chronic prostatitis/chronic pelvic pain syndrome.
}

\author{
PATERSON, C. and KENNEDY, C.
}

2020

This is the peer reviewed version of the following article: PATERSON, C. and KENNEDY, C. 2020.

Pharmacological interventions for treating chronic prostatitis/chronic pelvic pain syndrome. Research in nursing and health, 43(5), pages 548-549, which has been published in final form at https://doi.org/10.1002/nur.22027. This article may be used for non-commercial purposes in accordance with Wiley Terms and Conditions for Use of Self-Archived Versions. 


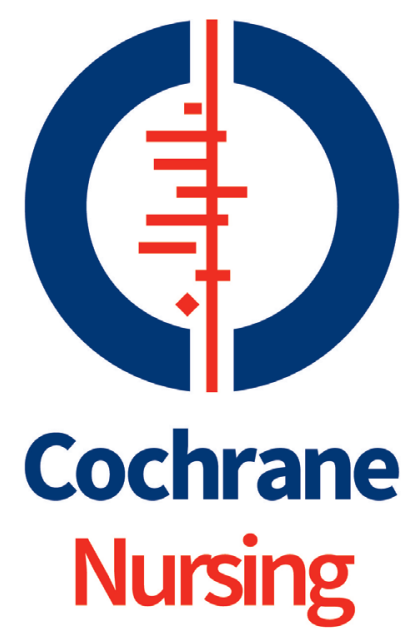

The Mission of Cochrane Nursing (CN) is to support Cochrane's work by increasing the use of their library and provide an international evidence base for nurses and related healthcare professionals involved in delivering, leading or researching nursing care. The $\mathrm{CN}$ produces 'Cochrane Corner' columns (summaries of recent nursing-care-relevant Cochrane reviews) that are regularly published in the group's collaborating nursing-care-related journals. The original authors and full citations of the Cochrane reviews are noted in each published column. These published summaries reach a much wider group of nurses and related healthcare agencies internationally and allow direct access to highly relevant evidence outcomes that frontline healthcare workers might not otherwise have access to. Information on the processes this group has developed can be accessed at: https://nursing.cochrane.org/

\section{Cochrane Nursing - Cochrane Review Summary}

\section{Prepared for the}

\section{Research and Nursing and Health Journal}

TITLE: Pharmacological interventions for treating chronic prostatitis/chronic pelvic pain syndrome 
Cochrane Corner Writer:

Catherine Paterson* PhD, BA, MSc, RAN, PG Cert LTA, FHEA

Associate Professor in Nursing

School of Nursing, Midwifery and Public Health, University of Canberra, ACT, Australia

Catherine.paterson@canberra.edu.au

Catriona Kennedy* PhD, BA (Hons) Dip Nurs. RN, DN PG Cert RNT

QNIS Professor of Community Nursing

School of Nursing and Midwifery, Robert Gordon University Aberdeen

Scotland.

\section{c.m.kennedy1@rgu.ac.uk}

${ }^{*} \mathrm{~A}$ member of the Cochrane Nursing $(\mathrm{CN})$

Background: Prostatitis is a common condition which can affect men of all ages; however, it is more frequently experienced in younger men with an initial onset around 40 years of age. The two main presenting clinical features of prostatitis are lower urinary tract symptoms and pelvic pain. Other symptoms might include obstructive or irritative voiding symptoms, ejaculatory pain, and haematospermia. A diagnosis is usually based on the man's history, physical examination, urinalysis and the two- or four-glass test (also known as obtaining urine specimens before, during, and after prostatic massage). Further investigations may also be performed when considering the differential diagnosis. Prostatitis can result in a significant reduction in quality of life (QOL) and pain can be associated with sexual dysfunction in men.

Prostatitis can be classified as four distinct types, namely: types I acute prostatitis, type II chronic bacterial prostatitis, type III chronic prostatitis/chronic pelvic pain syndrome (CP/CPPS), and type IV being asymptomatic prostatitis. It is unclear whether type III can be linked in all cases to prostatic involvement therefore the alternate denomination CPPS is used in clinical practice. Furthermore, CP/CPPS is further sub-classified as type IIla (inflammatory), and type IIIb (noninflammatory) which is dependent on the presence of inflammatory cells in prostatic secretions. CP/CPPS is considered when pelvic pain is present for at least three of the preceding six months with no other identifiable causes determined. 
There are several hypothesised causes of CP/CPPS which include: neuropsychological factors, infection, inflammation/autoimmunity and dyssynergic voiding associated with bladder neck hypertrophy. Given the wide range of potential causes of CP/CPPS there are many pharmacological interventions available in clinical practice to manage this complex condition. It is important to critically summarise evidence-based recommendations to inform the clinical management of men affected by CP/CPPS.

Objective/s: The overall aim was to assess the effects of pharmacological therapies for chronic prostatitis/chronic pelvic pain syndrome. The primary outcomes were prostatitis symptoms and adverse events. Secondary outcomes of interest were sexual dysfunction, quality of life, depression and anxiety and urinary symptoms. Participants in randomised controlled trials were men of all ages, suffering from chronic prostatitis/chronic pelvic pain syndrome (CP/CPPS) according to the type III classification.

Intervention/Methods: Studies compared individual pharmacological interventions which included: alpha blockers, 5-alpha reductase inhibitors, antibiotic therapy (quinolones, tetracyclines and other agents), anti-inflammatories (nonsteroidal antiinflammatory drugs [NSAIDs], corticosteroids), phytotherapy (pollen extract and bioflavonoids), botulinum toxin A, allopurinol, traditional medicine (traditional Chinese medicine, etc.), other pharmacological agents, or combination therapies head-tohead with placebo, no treatment or other types of pharmacological interventions. Comparator's included for example, alpha blockers plus quinolones versus placebo, or alpha blockers versus placebo. The researchers sought to determine whether individual or multimodal pharmacological interventions were more effective compared to the control in outcomes (prostatitis symptoms, adverse events, sexual dysfunction, QoL, depression and anxiety) measured up to and including 12 months after randomisation as short-term, and later than 12 months as long-term.

Results: Ninety-nine RCT studies and a total of 9119 participants, who were men with CP/CPPS, were included. The included studies conducted assessments of all available pharmacological interventions compared to placebo or in head-to-head comparisons. Sixteen pharmacological interventions were included and most comparisons were based on short-term follow-up (less than 12 months). Twenty-one studies were funded by pharmaceutical companies and the rest did not report funding sources. 
The reviewers were uncertain about the effects of Alpha blockers on prostatitis symptoms when compared to placebo at both short- and long-term follow-up. Alpha blockers may be associated with an increased incidence of adverse events, such as dizziness and postural hypotension. The findings suggest that Alpha blockers probably result in little to no difference in sexual dysfunction, quality of life and anxiety and depression.

The findings (based on moderate - low quality of evidence) suggest that antibiotics, anti-inflammatories, intraprostatic botulinum $\mathrm{A}$ injection, traditional Chinese medicine, mepartricin and phosphodiesterase inhibitors may improve prostatitis symptoms in the short term with limited adverse events. Moderate-quality evidence identified that phytotherapy and 5-alpha reductase inhibitors may improve prostatitis symptoms, but these interventions can be associated with a greater incidence of decreased libido, fatigue, mood change and gastrointestinal discomfort.

Conclusions: The authors of this review conclude there exists mainly low- to very low-quality evidence that alpha blockers, antibiotics, 5-ARI, anti-inflammatories, phytotherapy, intraprostatic BTA injection, and traditional Chinese medicine may reduce prostatitis symptoms without increased incidence of adverse events in the short term. This excludes alpha blockers which may be associated with an increase in mild adverse events. Few trials have been conducted with active comparators and therefore little evidence exists of the effects of these drugs on sexual dysfunction, quality of life or anxiety and depression. More rigorous research is required as the current evidence base offers little guidance for nurses as to effective pharmaceutical interventions for CP/CPPS.

Future research should ensure adequate study design, sample size and follow up longer than 12 weeks. Overall uncertainty about the true effects of a range of available interventions evidence in this review means we can have limited confidence in these results for clinical decision-making. Treatment decisions about the appropriate pharmacological intervention should be aligned to the symptoms being experienced, the safety profile of each agent and the patient's values and preferences.

Implications for Practice: CP/CPPS syndrome is a relatively common condition in men over the age of 40 years old. CP/CPPS is complex condition to treat and is debilitating for men which can negatively affect QOL. Nurses are a key member of 
the multidisciplinary team and can support patients by developing shared selfmanagement care plans based upon evidence-based recommendations identified in this review. There were varying levels of evidence across a wide range of pharmacological interventions to treat CP/CPPS which has informed clinical practice.

Importantly, nurses should be aware that there was low- to very low-quality evidence to support the use of alpha blockers with increased risk of hypotension and dizziness. Whereas other interventions including antibiotics, anti-inflammatories, intraprostatic botulinum A injection, traditional Chinese medicine, mepartricin and phosphodiesterase inhibitors may improve prostatitis symptoms with limited adverse events. Noteworthy, moderate-quality evidence identified that phytotherapy and 5alpha reductase inhibitors may improve prostatitis symptoms, but these interventions can be associated with a greater incidence of decrease libido, fatigue, mood change and gastrointestinal discomfort.

Nurses must ensure that patients are informed of the evidence-base recommendations for different pharmacological interventions to treat CP/CPPS and men must be carefully counselled about the potential adverse events which may further impact QOL.

\section{References:}

Franco JVA, Turk T, Jung JH, Xiao YT, lakhno S, Tirapegui Fl, Garrote V, Vietto V. Pharmacological interventions for treating chronic prostatitis/chronic pelvic pain syndrome. Cochrane Database of Systematic Reviews 2019, Issue 10. Art. No.: CD012552. DOI: 10.1002/14651858.CD012552.pub2. 